\title{
Von der Blutprobe bis zum Laborergebnis - Wie bekomme ich patientenorientiert das optimale Ergebnis?
}

\author{
Ruth Klein
}

\begin{abstract}
Laboruntersuchungen sind integraler Teil vieler tierärztlicher Untersuchungen. Der Anspruch an eine Laboruntersuchung ist es, die zugrunde liegende Fragestellung möglichst konkret zu beantworten. Dies ist unabhängig davon, ob die Probe im praxis- bzw. klinikeigenen oder aber in einem Versandlabor untersucht wird. Die Messergebnisse sollten die physiologische bzw. pathologische Situation des Tieres zum Zeitpunkt der Probennahme möglichst genau widerspiegeln. Der Beitrag informiert darüber, was es zu beachten gibt.
\end{abstract}

\section{Einleitung}

Biologisches Material wie Blut, Kot, Harn, Haare/Haut, Punktatmaterial oder aufwendig entnommenes Organmaterial verändert sich nach der Gewinnung. Es bedarf bis zur Messung der gewünschten Parameter unterschiedlicher Aufarbeitung und Lagerung.

Die Aussagekraft der Laborergebnisse wird durch bestimmte Faktoren beeinflusst:

- Nahrungsaufnahme

- bestehende Medikation

- Entnahmesituation

- gewonnenes Probenvolumen

- Aufarbeitung und Lagerung der Probe

Zuletzt entscheidet der Patientenbesitzer, der für die entstehenden Kosten aufkommen muss, über den Umfang der Untersuchung.

\section{Indikationen}

Die Gründe für eine Probenentnahme sind vielfältig, einer der häufigsten ist die Bestätigung einer anhand der klinischen Symptomatik und Voruntersuchung gestellten Verdachtsdiagnose. Daneben nehmen Therapiekontrollen, prä-OP-Scree- nings, regelmäßige jährliche Gesundheitschecks sowie Untersuchungen auf Reisekrankheiten nach einem Auslandsaufenthalt einen großen Raum ein.

\section{Optimieren der Proben}

\section{Probenentnahme}

Geplante Blutentnahmen stellen in der Patientenvorbereitung in der Tiermedizin zumeist kein Problem dar. Je nach Fragestellung wird der Patient nüchtern oder eine bestimmte Zeit nach Medikamentengabe einbestellt. Beim Harn wird meist Morgenurin gewonnen.

\section{.konkret}

Körperliche Anstrengung sollte vor einer Probenentnahme vermieden werden; hier gilt es, den Tierhalter entsprechend aufzuklären.

Sollten die optimalen Rahmenbedingungen nicht eingehalten werden, müssen die dadurch verursachten Messwertänderungen bei der Interpretation der Ergebnisse berücksichtigt werden. Viele klinisch-chemische Parameter verändern sich zeitabhängig nach der Nahrungsaufnahme. Die Ergebnisse können dann vorübergehend außerhalb der Normbereiche liegen, ohne hinweisend auf eine Erkrankung zu sein. In > Tab. 1 sind einige alternative Parameter aufgelistet, wenn der Einfluss der Nahrungs- aufnahme auf den zu bestimmenden Messwert möglichst gering gehalten werden soll.

\section{Probenvolumen}

Aus einem Kleinsäuger kann auch mit größten Bemühungen keine Dogge werden.

\section{Bei begrenztem Probenvolumen sollte} die auftraggebende Praxis dem Labor von vornherein eine Prioritätenliste mitgeben.

Die Untersuchungen können dann je nach Priorität für das Tier durchgeführt werden. Hier ist eine sinnvolle Kombination von Parametern zur Abklärung einer oder mehrerer Symptome zu wählen, statt die klassische Abklärung über mehrere Parameter pro Organsystem durchzuführen.

\section{Art des Probenmaterials}

Die Art des Probenmaterials kann bei zu erwartenden geringen Mengen entsprechend gewählt werden. Zumeist ist die Entnahme von Serum und EDTA für ein Organscreening erforderlich. Dies ist bei Problempatienten nur begrenzt möglich. Die Alternative ist Lithium-HeparinBlut. Aus Heparin-Plasma sind die meisten Parameter gleichwertig oder nur mit geringen Einschränkungen zu messen. Aus dieser Probe kann im 1. Schritt das Blutbild gemessen werden, nach Zentrifugation können die klinisch-chemische

Tab. 1 Alternativen zu Nüchternparametern.

\begin{tabular}{lll}
\hline Parameter & Alternative & Einschränkung \\
\hline Glukose & $\begin{array}{l}\text { - Fructosamine } \\
\text { - Harnstatus }\end{array}$ & nicht für Tagesprofile geeignet \\
\hline TLI & pankreatische Elastase (Kot) & nicht bei der Katze möglich \\
\hline Harnstoff & SDMA/Kreatinin & - \\
\hline
\end{tabular}

TLI: Trypsin-like Immunoreactivity; SDMA: symmetrisches Dimethylarginin 
Parameter bestimmt werden. Die Ausbeute ist zudem bei Plasma bezogen auf die Probenmenge größer als bei Serum.

\section{Wahl der Parameter}

Häufig abzuklärende Symptome sind z.B. Polyurie/Polydypsie, Adipositas, Abmagerung, Polyphagie, Fieber, Juckreiz, Störungen im Bewegungsapparat wie auch Leistungsinsuffizienz. Zusammen mit der Anamnese und der klinischen Untersuchung werden Leitparameter für Organsysteme ( $\triangleright$ Tab.2) zusammengestellt, mit denen es möglich ist, die primäre Ursache zu identifizieren oder einzugrenzen. Tierartliche Unterschiede in der Kombination dieser Parameter müssen berücksichtigt werden.

Bei geringem Probenvolumen oder fehlender Compliance der Patientenbesitzer kann über eine Stufen- und Ausschluss- diagnostik eine materialsparende und kostenoptimierte Labordiagnostik durchgeführt werden. In Anlehnung an > Tab. 2 werden die Messparameter zusammengestellt. Nach Ermittlung erster Ergebnisse können, wenn nötig, weitere auf ein Organsystem begrenzte Parameter nachgemessen werden. Liegt von der Probe noch Material vor, ist die Folgeuntersuchung ohne zusätzliche Entnahme möglich. Bringt die Erstuntersuchung keinen Hinweis auf die Primärerkrankung, kann versucht werden, über den Ausschluss der abgeklärten Organsysteme die Ursache einzugrenzen. Es wird nach Reihenfolge der wahrscheinlichsten Diagnose untersucht, bis eine der Verdachtsdiagnosen bestätigt ist. Das Wissen über die Interaktion der Organsysteme und die daraus resultierende Symptomatik ist Voraussetzung.

\section{Medikamentengabe}

Viele Patienten stehen unter Medikation, wenn eine Probenentnahme ansteht. Diese kann aus medizinischen Gründen nicht immer unterbrochen werden. Wichtig ist, ob die Medikation die Blutkonzentrationen der zu messenden Parameter verändert und somit eine echte Nebenwirkung ist oder ob es zu Interferenzen der Substanzen mit dem verwendeten Messsystem kommt. Beispiel für Letzteres ist die Kreatininbestimmung mittels der Methode nach Jaffé. Hier kommt es nach Aufnahme von Vitamin C oder Barbitursäure zu erhöhten Messwerten. Beide Fälle müssen bei der Interpretation der Messwerte berücksichtigt werden. Bei Bedarf sollte vorab bei praxiseigenen Geräten beim Hersteller oder sonst beim Einsendelabor nachgefragt werden.

Tab. 2 Leitsymptome und zur Abklärung bestimmte klinisch-chemische Parameter.

\begin{tabular}{|c|c|c|c|c|}
\hline Leitsymptom & Organsystem & $\begin{array}{l}\text { Laborwerte Serum } \\
\text { Hund }\end{array}$ & $\begin{array}{l}\text { Laborwerte Serum } \\
\text { Katze }\end{array}$ & $\begin{array}{l}\text { Untersuchung aus alter- } \\
\text { nativem Material }\end{array}$ \\
\hline Polyurie/Polydypsie & $\begin{array}{l}\text { - } \text { Niere } \\
\text { - Leber } \\
\text { - } \text { Diabetes mellitus }\end{array}$ & $\begin{array}{l}\text { - } \text { SDMA } \\
\text { - } \text { ALT } \\
\text { - Fructosamine }\end{array}$ & $\begin{array}{l}\text { - } \text { SDMA } \\
\text { - } \text { ALT } \\
\text { - Fructosamine }\end{array}$ & $\begin{array}{l}\text { Harn: UPC, Status, } \\
\text { Sediment }\end{array}$ \\
\hline Abmagerung & $\begin{array}{l}\text { - } \text { Pankreas } \\
\text { - Niere } \\
\text { - Schilddrüse } \\
\text { - } \text { Diabetes mellitus } \\
\text { - Magen/Darm }\end{array}$ & $\begin{array}{l}\text { - } \text { TLI } \\
\text { - SDMA } \\
\text { - Fructosamine } \\
\text { - Elektrophorese }\end{array}$ & $\begin{array}{l}\text { - TLI } \\
\text { - SDMA } \\
\text { - Fructosamine } \\
\text { - } \mathrm{T4} \\
\text { - Elektrophorese }\end{array}$ & $\begin{array}{l}\text { - Harn: UPC } \\
\text { Kot: Parasiten, } \\
\text { pankreatische Elastase }\end{array}$ \\
\hline Fieber & $\begin{array}{l}\text { - Infektion } \\
\text { - Tumor } \\
\text { - Entzündung } \\
\text { - Autoimmunerkrankung } \\
\text { - Hyperthyreose }\end{array}$ & $\begin{array}{l}\text { - Blutbild } \\
\text { - Elektrophorese }\end{array}$ & $\begin{array}{l}\text { - Blutbild } \\
\text { - Elektrophorese } \\
\text { - T4 }\end{array}$ & $\begin{array}{l}\text { Harn: UPC, Status, } \\
\text { Sediment }\end{array}$ \\
\hline Anämie, Ikterus & $\begin{array}{l}\text { - Infektion } \\
\text { - Blutung } \\
\text { - Leber } \\
\text { - } \text { Autoimmunerkrankung }\end{array}$ & $\begin{array}{l}\text { - } \text { Blutbild } \\
\text { - Retikulozyten } \\
\text { - Elektrophorese } \\
\text { - } \text { ALT }\end{array}$ & $\begin{array}{l}\text { - } \text { Blutbild } \\
\text { - Retikulozyten } \\
\text { - Elektrophorese } \\
\text { - } \text { ALT }\end{array}$ & $\begin{array}{l}\text { - Blutausstrich } \\
\text { - Harn: Status, Sediment } \\
\text { - Kot: Parasiten, Hämokult }\end{array}$ \\
\hline Leistungsschwäche & $\begin{array}{l}\text { - } \text { Herz } \\
\text { - } \text { metabolische Störung } \\
\text { - } \text { Elektrolytverschiebung } \\
\text { - neuromuskuläre Erkrankung }\end{array}$ & $\begin{array}{l}\text { - Blutbild } \\
\text { - cTPI } \\
\text { - Kalzium } \\
\text { - Kalium } \\
\text { - Magnesium } \\
\text { - Elektrophorese }\end{array}$ & $\begin{array}{l}\text { - Blutbild } \\
\text { - cTPI } \\
\text { - Kalzium } \\
\text { - Kalium } \\
\text { - Magnesium } \\
\text { - Elektrophorese } \\
\text { - FeLV } \\
\text { - FIV }\end{array}$ & - \\
\hline Juckreiz/Haut & $\begin{array}{l}\text { - Infektion } \\
\text { - Autoimmunerkrankung } \\
\text { - Allergie } \\
\text { - metabolische Störung }\end{array}$ & $\begin{array}{l}\text { - Sarkoptes-Antikörper } \\
\text { - Allergie-Screening }\end{array}$ & Allergie-Screening & $\begin{array}{l}\text { - Biopsie } \\
\text { - Abstrich für BU und PCR }\end{array}$ \\
\hline
\end{tabular}


Tab. 3 Alternatives Probenmaterial zu Blutuntersuchungen.

\begin{tabular}{|ll}
\hline Organsystem/Indikation & Alternativen zur Blutuntersuchung \\
\hline $\begin{array}{ll}\text { Niere/Nebenniere (Nieren- } \\
\text { insuffizienz, Endokrinopathien) }\end{array}$ & $\begin{array}{l}\text { Untersuchung von } \\
\text { - Harn (Eiweiß-Kreatinin-Quotient, Kortisol, Katechol- } \\
\end{array}$ \\
& amine) \\
& Speichel (Kortisol) \\
Magen-Darm-Trakt & Kot (Bakteriologie, Parasitologie, chemische Unter- \\
& suchungen) \\
& Harn (Stoffwechselprodukte) \\
Mineralstoffversorgung & Haare
\end{tabular}

Ein besonderes Problem stellt die Gabe von Kortikosteroiden dar, die neben einer kurzfristigen Wirkung auf Elektrolyt-, Mineralstoff- und Eiweißhaushalt eine Langzeitwirkung auf das Immunsystem zeigt. Sollte ein Absetzen nicht möglich sein, ist z.B. eine Antikörperbestimmung nicht zielführend. Alternativ sollte bei einem Infektionsgeschehen auf den Erregerdirektnachweis zurückgegriffen werden.

\section{Einfluss des Patienten auf die Laborergebnisse}

Blutproben, die von extrem ängstlichen Tieren oder nur unter großem Stress gewonnen werden können, sind in ihrer Aussagekraft in vielen Parametern eingeschränkt. Vor allem die Muskelparameter (CK, LDH, AST), das Blutbild (Stressleukogramm), Hormone wie die Schilddrüsenhormone T4, fT4 und die Nebennierenhormone Kortisol, Aldosteron und die Katecholamine lassen häufig keinen Rückschluss mehr auf die Grunderkrankung zu. Bei sehr kachektischen Tieren ändert sich aufgrund der geringen Muskelmasse die Interpretation einiger Parameter. Hier verlieren CK, Kreatinin und LDH an Aussagekraft. Auch längere Stauungszeiten sind problematisch, da sie u.a. zur Freisetzung von Gewebsthromboplastin (Gerinnungsaktivierung) und zur Zunahme von Kalium (Hämokonzentration durch Flüssigkeitsaustritt ins Interstitium) führen.

\section{.konkret}

Venen sollten für die Blutentnahme nur etwa 30 Sekunden und möglichst nicht länger als 3 Minuten gestaut werden.

Alternative Probenmaterialien ( $\triangleright$ Tab.3) unterliegen bei einigen Parametern nicht kurzfristigen Einflussfaktoren wie Stress oder Angst, auch Stauung spielt dann keine Rolle. Diese Proben können unter Umständen sogar vom Besitzer in einer stressärmeren Situation gewonnen werden.

\section{Fazit}

Es ist in vielen Fällen möglich, bei nicht optimalen Bedingungen aus wenig Probenmaterial oder bei schwierigen Patienten über die Auswahl des richtigen Probenmaterials und der zu bestimmenden Parameter eine zielgerichtete Laboruntersuchung auch kostenoptimiert durchzuführen.

\section{Online zu finden unter}

https://doi.org/10.1055/s-0043-110389

\section{Dr. Ruth Klein}

Laboklin GmbH \& Co KG

Steubenstr. 4

97688 Bad Kissingen

klein@laboklin.de 